\title{
A laminar flow unit for the care of critically ill newborn infants
}

This article was published in the following Dove Press journal:

Medical Devices: Evidence and Research

17 October 2013

Number of times this article has been viewed

\section{Jose MR Perez' \\ Sergio G Golombek ${ }^{2}$ \\ Carlos Fajardo 3 \\ Augusto Sola ${ }^{4}$}

'Stella Maris Hospital, International Neurodevelopment Neonatal Center (CINN), Sao Paulo, Brazil; ${ }^{2}$ M Fareri Children's Hospital, Westchester Medical Center, New York Medical College, Valhalla, NY, USA; ${ }^{3}$ University of Calgary, Calgary, Canada; ${ }^{4}$ St Jude Hospital, Fullerton, California, CA, USA

Video abstract

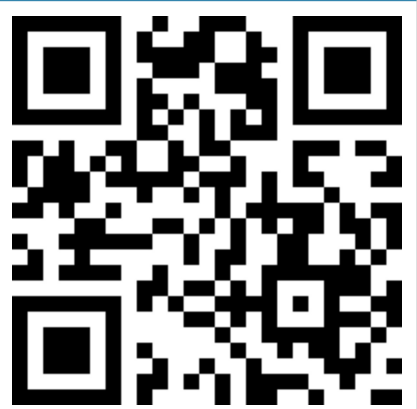

Point your SmartPhone at the code above. If you have a QR code reader the video abstract will appear. Or use: http://dvpr.es//chG9uK
Correspondence: Augusto Sola 24896 Sea Crest Dr,

Dana Point, CA, 92629, USA

Tel +l 9494338962

Email augustosolaneo@gmail.com
Introduction: Medical and nursing care of newborns is predicated on the delicate control and balance of several vital parameters. Closed incubators and open radiant warmers are the most widely used devices for the care of neonates in intensive care; however, several well-known limitations of these devises have not been resolved. The use of laminar flow is widely used in many fields of medicine, and may have applications in neonatal care.

Objective: To describe the neonatal laminar flow unit, a new equipment we designed for care of ill newborns.

Methods: The idea, design, and development of this device was completed in Sao Paulo, Brazil. The unit is an open mobile bed designed with the objective of maintaining the advantages of the incubator and radiant warmer, while overcoming some of their inherent shortcomings; these shortcomings include noise, magnetic fields and acrylic barriers in incubators, and lack of isolation and water loss through skin in radiant warmers. The unit has a pump that aspirates environmental air which is warmed by electrical resistance and decontaminated with High Efficiency Particulate Air Filter (HEPA) filters (laminar flow). The flow is directed by an air flow directioner. The unit has an embedded humidifier to increase humidity in the infant's microenvironment and a servo control mechanism for regulation of skin temperature.

Results: The laminar flow unit is open and facilitates access of care providers and family, which is not the case in incubators. It provides warming by convection at an air velocity of $0.45 \mathrm{~m} / \mathrm{s}$, much faster than an incubator $(0.1 \mathrm{~m} / \mathrm{s})$. The system provides isolation 1000 class (less than 1,000 particles higher than 0.3 micron per cubic feet at all times). This is much more protection than an incubator provides and more than radiant warmers, which have no isolation whatsoever. Additionally, it provides humidification of the newborn's microenvironment (about $60 \%$ relative humidity), which is impossible with a radiant warmer, which produces high water body loss. It has no mechanical barriers like acrylic walls, its magnetic field is lower than an incubator $(0.25 \mu \mathrm{t}$ versus $1.2 \mu \mathrm{t})$, and the noise is minimal compared to incubators. The unit is also able to provide controlled total body hypothermia, which is not possible with either of the other two units.

Conclusion: The laminar flow unit for neonatal care is a novel device which we recently developed. The introduction of laminar flow technology represents a real innovation in the neonatal field. We have described the various components of the unit and the potential advantages for management of ill neonates. This will hopefully lead to improved clinical outcomes and more effective neonatal management and safety.

Keywords: laminar flow, newborn intensive care, incubator, radiant warmer

\section{Introduction}

The medical care of newborns is based on the delicate control of several vital parameters after stabilization in the delivery room. The major parameters that need to be checked 
and well-maintained are body temperature, ventilation, oxygenation, hydration, and level of bacterial contamination. In an attempt to provide better temperature control, all sick newborn infants are cared for in units that have been expressly designed to provide them with a unique microenvironment. These units are the incubator and the radiant warmer.

The first incubator was developed in 1880 by Tarnier and Couney. ${ }^{1}$ The ground-breaking innovation was the idea of surrounding a newborn with humidity and warm air. Despite innovation and an explosion in new technology in the last 3 to 4 decades, the original concept has not changed much in the last 100 years. Old and well-known problems have also not been resolved, including the spatial limitations of treating a sick newborn inside of an enclosed environment, the changes in temperature and humidity when an incubator is opened, the difficulty in achieving contact for the mother, and a lower capacity to maintain temperature compared with a newer type of unit, the radiant warmer. ${ }^{2}$ In incubators, temperature is regulated by convection. The difficulty in maintaining temperature is explained by the low air velocity inside an incubator (approximately $0.1 \mathrm{~m} / \mathrm{s}$ ), which results in a low convection heat transmission, ${ }^{3,4}$ and probably involved transferring some heat by radiation rather than convection. Another problem is the noise inside the incubator ${ }^{5,6}$ which may be as high as $100 \mathrm{~dB} \cdot{ }^{6}$ Additionally, it has been found that newborn infants who are treated in incubators for more than several days can be exposed to high level magnetic fields. ${ }^{7,8}$ Such magnetic fields pose come potential risks, including leukemia, ${ }^{9}$ and while these risks would be lower if the incubator's engine was protected, this is not the case in the majority of incubators.

In the 1970 s an alternative to the incubator was launched in the market - the radiant warmer. This equipment has some advantages, such as easy accessibility for care, lower manufacturing costs, and lower maintenance cost. Unfortunately, this open unit is unable to provide a low bacteria level environment, as its warmth is transmitted through radiation. This is a problem because warmth through radiation may lead to a high water loss, particularly in premature newborns. ${ }^{10}$ A meta-analysis trial comparing incubator and radiant warmer $^{2}$ in 2009 confirmed the higher water loss associated with the latter but did not find definitive answers on which one was the superior equipment.

Laminar flow occurs when a fluid flows in parallel layers, with no disruption between the layers. There are no cross currents perpendicular to the direction of flow, nor any eddies or swirls in the fluid. In laminar flow, the motion of the particles of the fluid is very orderly, with all particles moving in parallel straight lines. Laminar flow occurs at low Reynolds numbers, where viscous forces are dominant, and it is characterized by smooth and constant fluid motion. ${ }^{11}$ The use of laminar flow in medicine started in the 20th century. Laminar flow has been shown to decrease contamination and infection rates, and as a result it is widely used in medicinal applications. ${ }^{12-21}$ The benefits of laminar flow have been shown in the preparation of intravenous nutrition in order to reduce the risk of contamination, as well as in surgery suites and intensive care units, where they have been used to protect the environment from contamination. ${ }^{12,13,15-17}$ It has also been demonstrated that warm laminar flow does not increase the risk of bacterial growth; ${ }^{14,18}$ Laminar flow also has an influence on prosthetic joint infections, ${ }^{19}$ and it was recently shown to reduce cases of surgical site infections in vascular patients. ${ }^{20}$

Laminar flow technology has therefore become an asset in many areas of medical care; indeed, it has successfully been used for diverse clinical applications since the 1980s. ${ }^{12-20}$ However, its use in neonatology is still very limited.

Our objective is to describe the neonatal laminar flow unit, a new device for care of ill newborns recently developed in Brazil. We named this device the NeoNatFlow, as it maintains the advantages of closed incubators and radiant warmers while overcoming some of their inherent issues by applying the laminar flow's technology to neonatal care.

\section{Methods}

The idea, design, and development of this device was completed in Sao Paulo, Brazil. The unit is an open mobile bed planned with the objective of maintaining the advantages of the incubator and open radiant warmer while overcoming some of their inherent problems. For incubators, we looked to address difficulty in accessing a critically ill infant, excessive noise, magnetic fields and acrylic barriers; in radiant warmers, we targeted radiation source of heat, the lack of humidity, and increased water loss through the skin and absence of isolation. Additionally, the system had to be able to normalize temperature in babies with hypothermia in a controlled but swift way, while at the same time being able to easily maintain temperatures in the normal range. We worked with bioengineers for over 3 years and developed several prototypes until we reached the final stage, which is described below.

\section{Laminar flow unit}

The unit is an open mobile bed. It has a pump that aspirates environmental air, producing a noise measured at $50 \mathrm{~dB}$ on 
over 15 different occasions using a digital decibel meter (digital decibel meter with Leq ITDEC-3000; Instrutemp Instrum Medição Ltda, Sao Paulo, Brazil); this is significantly less than the noise produced by incubators, which may on occasion be as high as $100 \mathrm{~dB}$. The unit's magnetic field was measured (MFM10, Combinova AB, Domkraftsvägen, Sweden) consistently as $0.25 \mu \mathrm{t}$; this is markedly lower than an incubator's magnetic field, which is reported as $1.2 \mu \mathrm{t}$.

The air that is drawn is decontaminated by passing through a High Efficiency Particulate Air Filter (HEPA) which filters particles as small as 0.3 microns with a $99.97 \%$ minimum particle-collective efficiency filters. The air is then blown in a very smooth laminar flow directed by an air flow directioner made of acrylic. With the exact angle developed in the acrylic directioner, the laminar air flow covers the entire area where the newborn lies, and there is no increase in particles seen at the foot end of the unit. This is proof that there is no contamination with ambient air, and that there is laminar flow and not lack of flow or turbulent flow at that site. In order to achieve this, we went through different steps in the development phases. The classic acrylic "barrier" (or wall) at the foot end of the unit can (and did) disrupt the flow when we did initial testing in the first prototype. We then repeated testing leaving the end wall down (not raised) or removing it, and the problem was solved. However, we did not consider this to be a safe way to care for babies in the unit. Therefore, we developed an acrylic "barrier" or wall with holes in it. We tested this setup (AEROTRACK 9350, Sao Paulo, Brazil) using 30 different carefully designed experiments and found that there were never more than 600-700 particles per cubic feet at the foot end, the same amount found in the head end and in the middle of the area where the infant lies. Therefore we elected to equip the unit with a foot end "wall" with holes. After the foot end of the unit, laminar flow disappears. The system provides isolation Class 1000 of the International Standard Organization, or ISO 4 (less than 1,000 particles larger than 0.3 microns per cubic foot) of the Federal Standard 209E. In summary, in this unit the protection against contamination is twofold - the HEPA filters air flow and the laminar flow of the filtered air provides a surface which is free of large particles, even during handling and procedures that ill neonates need to survive.

The air is warmed by a heater with an electric resistance (Novus $^{\circledR}$; KMI Novus, GoGaS Goch GmbH \& Co. KG, Dortmund, Germany), which converts electrical energy into heat. Heat is provided by convection in a silent manner, with minimal risk of ignition hazard compared to radiant electric heaters. The air velocity is $0.45 \mathrm{~m} / \mathrm{s}$ and a servo control mechanism increases or lowers heat emission to regulate the newborn's skin temperature. The air is humidified through an embedded humidifier to increase humidity in the infant's microenvironment to a maximum of $60 \%$ relative humidity.

The NeoNatFlow is therefore an open unit that has no mechanical barriers (eg, the acrylic walls of an incubator) and provides isolation-humidity and heat by convection and not by radiation, making it different from the open radiant warmer. Figure 1 shows all components of the NeoNatFlow unit schematically. Figure 2 is a photograph of a current prototype of the NeoNatFlow.

An additional and unique feature of this equipment is that it is also able to provide supervised total body hypothermia using convection and servo-controllers to maintain the desired low body temperature, which cannot be provided by an incubator or radiant warmer. Hypothermia by other methods is a proven therapy for perinatal hypoxic brain injury.

\section{Discussion}

We have described the properties of a newly developed unit with laminar flow for the care of ill newborns. It is an innovation that may be proven to be better for neonatal microenvironment and care than the existing technologies. The goal of our team over the course of several years was to develop a unit for neonatal care which provided greater accessibility to the newborn, for

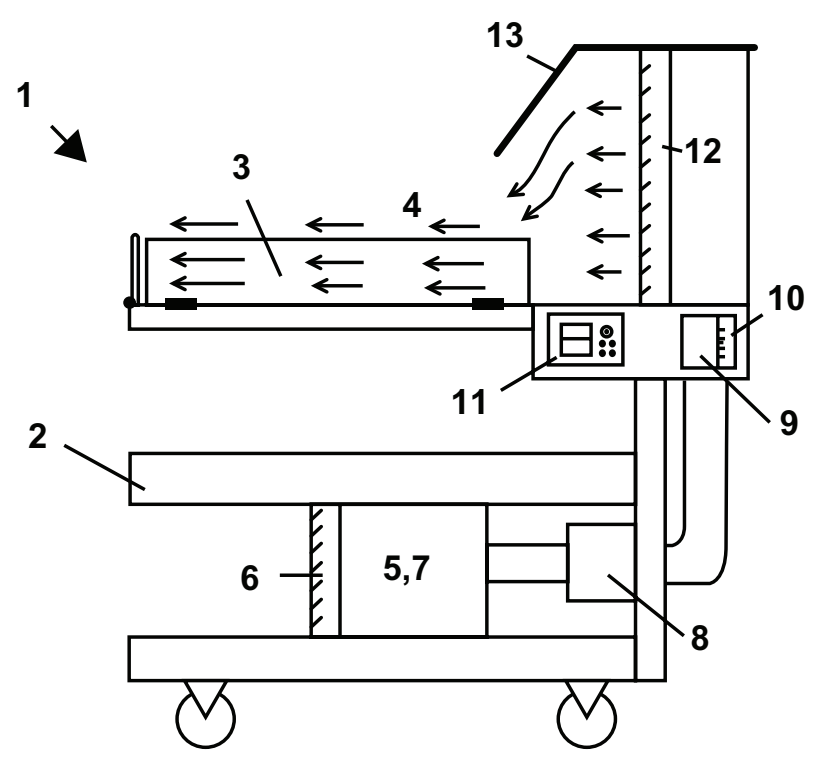

Figure I Schematic representation of the laminar flow unit for newborn care. Notes: (I) Laminar flow neonatal unit; (2) mobile equipment; (3) open bed; (4) laminar flow with control of temperature and humidity; (5) engine that aspirates environmental air; (6) air warmed by electrical resistance; (7) engine; (8) servo control equipment; humidifier; (I0) water level by humidifier; (II) temperature control; ( I 2) HEPA filter; (13) guiding of air flow.

Abbreviation: HEPA, High Efficiency Particulate Air. 


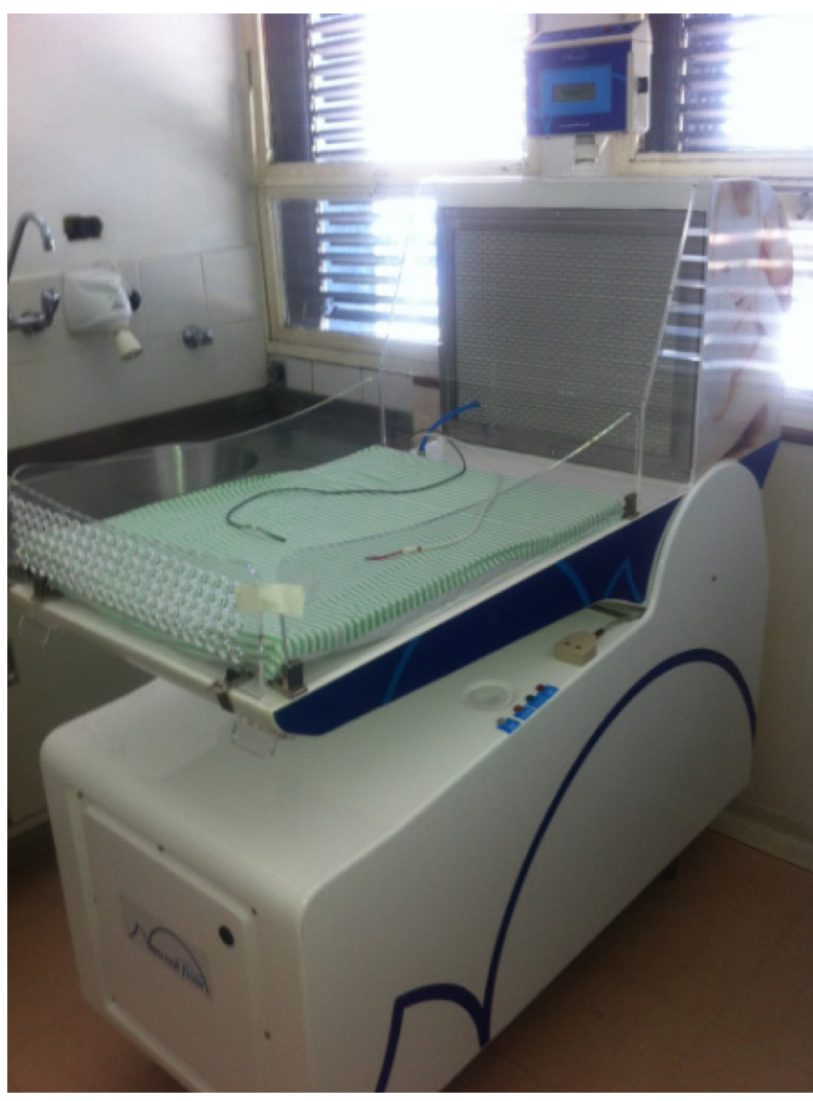

Figure 2 Prototype of the Laminar Flow Unit.

both the care team and the baby's family, while at the same time maintaining isolation and humidity. The laminar flow system may become the solution that achieves these goals.

Laminar flow has been used in medicine for almost 40 years, but its use in neonatology is still very limited. To our knowledge, this is the first description of a laminar flow unit designed for neonatal care. During development, some challenges arose, including the need to add warm air and humidity to the laminar flow. Additionally, some pleasant surprises arose; for example, the relatively low manufacturing costs associated with this technology is much lower than an incubator and similar to a radiant warmer. The maintenance cost should also be much more inexpensive because, compared with incubators which require filter replacements every 3 months, the HEPA filter has to be changed only once a year in our prototype.

After developing and improving prototypes of this laminar flow unit, we had several questions that needed to be evaluated. They pertain to, among other things, the time to normalize temperature in babies with hypothermia, the ability to maintain temperature in the normal range, and rates of skin colonization, water balance, and hydration. After obtaining informed consent approved by Ethical Committees of the Saúde Guarulhos
Hospital, we completed some proof of concept clinical studies on these topics and are ready to submit them for publication. Some preliminary findings have been presented at international meetings, ${ }^{21,22}$ but have not been yet peer-reviewed. In summary, we found that this equipment was able to increase temperature in babies with hypothermia faster than an incubator. The air velocity in this equipment transfers heat more quickly than an incubator (prototype air velocity: $0.45 \mathrm{~m} / \mathrm{s}$; incubator air velocity: $0.1 \mathrm{~m} / \mathrm{s}$ ) but may also increase body heat losses, which is a potential first limitation. Another potential limitation is that the NeoNatFlow can deliver $60 \%$ humidity. Even though this is significantly more than radiant warmers, which have no humidity whatsoever, it is less than what is desired by many clinicians, and lower than what can be delivered by some modern incubators. ${ }^{23,24}$ However, in some cases this potentially highly humid environment is offset by the frequent opening of incubator windows. In reality, most unstable infants are treated in radiant warmers for improved accessibility, sacrificing all humidity in the microenvironment. In one of our preliminary studies, ${ }^{21}$ we assessed water balance and urinary output, since open radiant warmers have been previously shown to produce significant loss of insensible water. This did not occur with the laminar flow unit, likely due to its embedded humidifier, a feature not present in radiant warmers.

The neonatal laminar flow unit could potentially be used in the delivery room just like radiant warmers are currently being used, but with the added advantages of isolation and humidification. This unit was also able to maintain neonatal temperature in the normal ranges, as well as guaranteeing low skin colonization in term, near term, and moderately preterm newborns. Another potential use is as a surgical bed, with the advantage of a lower impact on the medical team due to the high ambient temperatures produced by radiant warmers, not produced at all by this lamnar flow unit.

Neonatal infection, especially bacterial infection, is a serious problem in all neonatal intensive care units in the world; it is the largest cause of mortality in developing countries. In Brazil, infection rates of 15-30 newborns / 1000 days with a mortality of about $70 \%$ in some regions of the country have been reported. The isolation produced by the ISO 4 standard is almost like a barrier to germs that can be introduced when handling infants. In addition to decreasing colonization rates, this unit could be useful in decreasing nosocomial infections.

Finally, this equipment is also able to provide supervised, total-body, moderate hypothermia, which has been proven to be useful in the treatment of perinatal hypoxic brain injury. Laminar flow hypothermia is likely to be as effective as other methods which are more distressing. We also have a proof 
of concept study on this topic which will be submitted for publication in the near future.

\section{Conclusion}

The laminar flow unit for neonatal care is a novel device which we recently developed. We have described the various elements in the unit and the potential advantages for management of ill neonates. We consider that the introduction of laminar flow technology represents a real innovation in the neonatal field; it could provide solutions to some of the current issues encountered by closed incubators and open radiant warmers explained by Antonucci et al. ${ }^{26}$ Clinical studies are needed and are being performed; we speculate that this neonatal laminar flow unit could be at least part of the solution for some of the issues encountered while caring for newborns, particularly in areas of the world with limited access to very expensive technology. This will hopefully lead to improved clinical outcomes and more effective neonatal management and safety.

\section{Acknowledgments}

The authors are very thankful to anonymous Reviewer 2. Their insightful and positive comments and suggestions have helped us improve the manuscript significantly.

\section{Disclosure}

The authors report no conflicts of interest in this work. JMRP holds a patent of the Neonatal Laminar Flow Unit.

\section{References}

1. Baker JP. The incubator and the medical discovery of the premature infant. J Perinatol. 2000;20(5):321-328.

2. Gray PH, Flenady V. Cot-nursing versus incubator care for preterm infants. Cochrane Database Syst Rev. 2011;(8):CD003062.

3. McIntosh N, Lyon AJ. Keeping infants warm. Pediatrics. 2002; 110(2 Pt 1):424-425.

4. Apedoh A, el Hajajji A, Telliez F, Bouferrache B, Libert JP, Rachid A. Mannequin-assessed dry-heat exchanges in the incubator-nursed newborn. Biomed Instrum Technol. 1999 Sep-Oct;33(5):446-54.

5. Johnson AN. Neonatal response to control of noise inside the incubator. Pediatr Nurs. 2001;27(6):600-605.

6. Rodarte MDO, Scochi CGS, Leite AM, Fujinaga CI, Zamberlan NE, Castral TC. O ruído gerado durante a manipulação das incubadoras: implicações para o cuidado da enfermagem. Rev Latino Am Enfermagem. 2005;13(1):79-85. Portuguese.
7. Klauss MA, Fanaroff AA, editors. The Physical Environment. In Care of the High-Risk Neonate. 5th ed. Philadelphia: Saunders; 2001.

8. Bellieni CV, Bagnoli F, Pinto I, Stacchini N, Buonocore G. Reduction exposure of newborns and caregivers to very high electromagnetic fields produced by incubators. Med Phys. 2005;32(1):149-152.

9. Söderberg KC, Naumburg E, Anger G, Cnattingius S, Ekbom A, Feychting M. Childhood leukemia and magnetic fields in infant incubators. Epidemiology. 2002;13(1):45-49.

10. Kjartansson S, Arsan S, Hammarlund K, Sjörs G, Sedin G. Water loss from the skin of term and preterm infants nursed under a radiant heater. Pediatr Res. 1995;37(2):233-238.

11. Rott N. Note on the history of the Reynolds number. Annu Rev Fluid Mech. 1990;22(1):1-12.

12. Leach D. Use of laminar flow in manufacturing large volume parenteral products. Bull Parenter Drug Assoc. 1972;26(3):104-108.

13. Clark RE. Laminar flow for a cardiac operating room. AORN J. 1972;15(5):61-65.

14. Brock L. The importance of environmental conditions, especially temperature, in the operating room and intensive care ward. Br J Surg. 1975;62(4):253-258.

15. Sanderson MC, Bentley G. Assessment of wound contamination during surgery: a preliminary report comparing vertical laminar flow and conventional theatre systems. Br J Surg. 1976;63(6):431-432.

16. Pietri P, Alagni G, Pancrazio F. Use of laminar flow apparatus in vascular surgery. Minerva Chir. 1980;35(19):1493-502. Italian.

17. Thurston AJ. Of blood, inflammation and gunshot wounds: the history of the control of sepsis. Aust N Z J Surg. 2000;70(12):855-861.

18. Sharp RJ, Chesworth T, Fern ED. Do warming blankets increase bacterial counts in the operating field in a laminar flow theatre? J Bone Joint Surg Br. 2002;84(4):468-468.

19. Gastmeier P, Breier AC, Brandt C. Influence of laminar airflow on prosthetic joint infections: a systematic review. J Hosp Infect. 2012;81(2): 73-78.

20. Bosanquet DC, Jones CN, Gill N, Jarvis P, Lewis MH. Laminar flow reduces cases of surgical site infections in vascular patients. Ann R Coll Surg Engl. 2013;95(1):15-19.

21. Perez JMR, Sola A, Golombek S, Fajardo C. Comparative analysis, with evaluation by numeric score, between neonatal laminar flow unity, intensive care incubator and radiant warmer. ABSTRACT \#A369, presented in oral platform session. 10th World Congress of Perinatal Medicine, Punta del Este, Uruguay 2011.

22. Perez JMR, Sola A, Golombek S, Fajardo C. Breaking the incubator paradigm A comparative study of late preterm and term infants using a new device: the neonatal laminar flow unit. ABSTRACT \#657, Poster session. Pediatric Academic Societies Meeting Vancouver, 2010.

23. Sinclair L, Crisp J, Sinn J. Variability in incubator humidity practices in the management of preterm infants. $J$ Paediatr Child Health. 2009;45(9):535-540.

24. Deguines C, Décima P, Pelletier A, Dégrugilliers L, Ghyselen L, Tourneux P. Variations in incubator temperature and humidity management: a survey of current practice. Acta Paediatr. 2012;101(3): 230-235.

25. Filho NA, Junior JMS. Perinatologia Básica. 3rd ed. Rio de Janeiro: Guanabara Koogan, Brazil; 2006. Portuguese.

26. Antonucci R, Porcella A, Fanos V. The infant incubator in the neonatal intensive care unit: unresolved issues and future developments. J Perinat Med. 2009;37(6):587-598.
Medical Devices: Evidence and Research

\section{Publish your work in this journal}

Medical Devices: Evidence and Research is an international, peerreviewed, open access journal that focuses on the evidence, technology, research, and expert opinion supporting the use and application of medical devices in the diagnosis, treatment and management of clinical conditions and physiological processes. The identification of novel

\section{Dovepress}

devices and optimal use of existing devices which will lead to improved clinical outcomes and more effective patient management and safety is a key feature. The manuscript management system is completely online and includes a quick and fair peer-review system. Visit http://www. dovepress.com/testimonials.php to read real quotes from authors. 